\title{
A Phalangeal Osteoid Osteoma: A Case Report
}

\author{
Khalequezzaman $\mathrm{S}^{1}$, Saha $\mathrm{BK}^{2}$, Khan $\mathrm{BS}^{3}$, Kumar $\mathrm{P}^{4}$
}

\begin{abstract}
Osteoid osteoma is a benign bone forming tumor of the growing skeleton that is most often seen in young men. It represents by pain and radiologic appearance of a nidus surrounded by osteosclerosis that occurs mostly in long bones of the lower extremity. Occurrence of this tumor in the hand is an uncommon condition. A 35 year old right-hand-dominant man presented to our orthopedic and trauma OPD with an approximately 2 year history of right middle finger pain without any history of trauma. He consulted previously with a local doctor and had undergone $\mathrm{x}$ ray of the hand with no definitive diagnosis. Computed tomography at our institution was consistent with the diagnosis of osteoid osteoma. The patient was treated with surgical excision of the lesion without bone grafting. The diagnosis of osteoid osteoma was confirmed by histopathology. Follow up visit showed complete resolution of pain. In this case demonstrating that osteoid osteoma is an important differential diagnosis in patients with finger pain.
\end{abstract}

\section{Key words}

Osteoid osteoma, phalanx, tumor

\section{Introduction}

Osteoid osteoma is a benign osteoblastic tumor that Bergstrand first described in 1930. Jaffe described it in 1935 and was the first to recognize it as a unique entity. It is characterized by nocturnal pain and local tenderness. Osteoid osteoma is a benign disease process of bone that usually affects children, adolescents, and young adults with the majority of patients being between the ages of 10 and 25 years. ${ }^{1}$ It is the third most common benign tumor, comprising $12 \%$ of benign tumors and $3 \%$ of all tumors, and has a male to female ratio of $2: 1{ }^{2}$ Pain, worse at night and relieved by oral non-steroidal anti-inflammatory medications is the most typical symptom. ${ }^{3}$ Since osteoid osteoma is a vascular tumor, substances that cause vasodilatation, such as alcohol, may precipitate acute pain.

This tumor is most frequently seen in the metaphysis or diaphysis of long bones with half the cases involving the femur or tibia but it can affect the posterior element of the spine (10\%), hands or feet $(12 \%){ }^{3} \quad$ Radiograph of the affected long bone shows a lucent area less than $1.5 \mathrm{~cm}$ in diameter called nidus surrounded by a zone of reactive bone formation in the cortical or medullary canal. ${ }^{4}$ Osteoid osteomas of the hand are uncommon, most commonly seen in the phalanges and often result in atypical clinical and radiologic findings. So, long delay in diagnosis is common since clinical findings may 


\section{CASE REPORT}

mimic a variety of diseases in differential diagnosis.

\section{Case Report}

A 33 year-old right hand dominant man presented to Orthopedic and Trauma OPD with pain in the middle finger of right hand for about 2 years. There is no history of trauma to the area. The pain had progressively increased in severity for the last couple of months and became unbearable especially at night. However, it usually relieved with oral NSAID. At the time of presentation, there was restricted movement of the affected finger. Radiographs done at the local area reported as normal. Physical examination

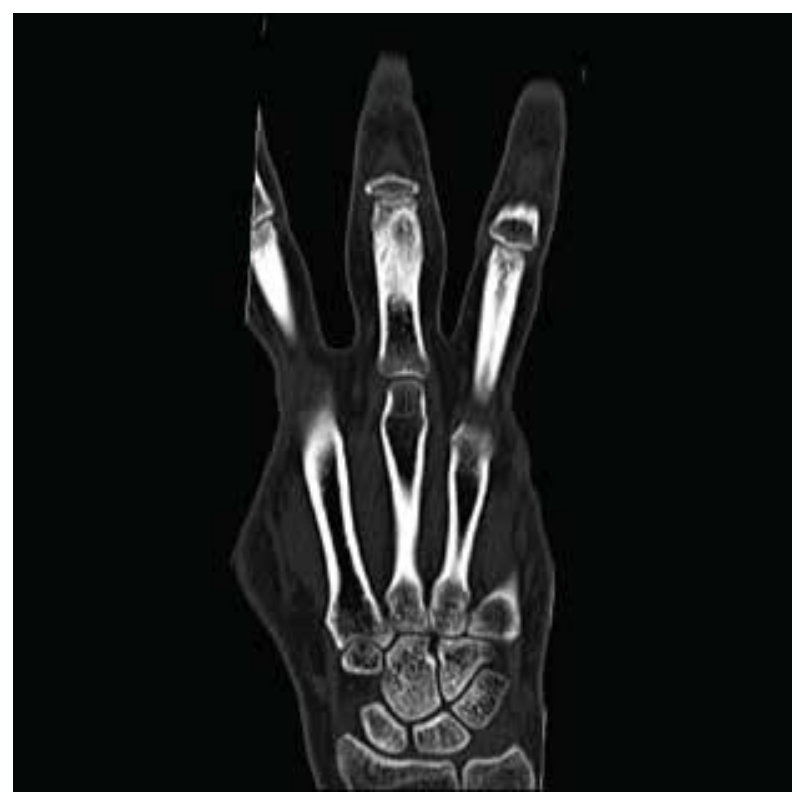

Fig.1 (a) demonstrated slight swelling and tenderness on the ulnar aspect of the proximal phalanx of the right middle finger. There was no erythema or induration. Neurovascular exam of the left upper extremity was normal.

He was ordered computed tomography (CT) scan of the right hand. This showed a focal lucent lesion at the ulnar aspect near the head of the proximal phalanx. The lesion measured 5.2 to 6.5 $\mathrm{mm}$ in diameter with no periosteal bone formation. A central nidus was present and surrounding sclerosis in the adjacent bone (Fig 1). These features are in consistence of an osteoid osteoma.

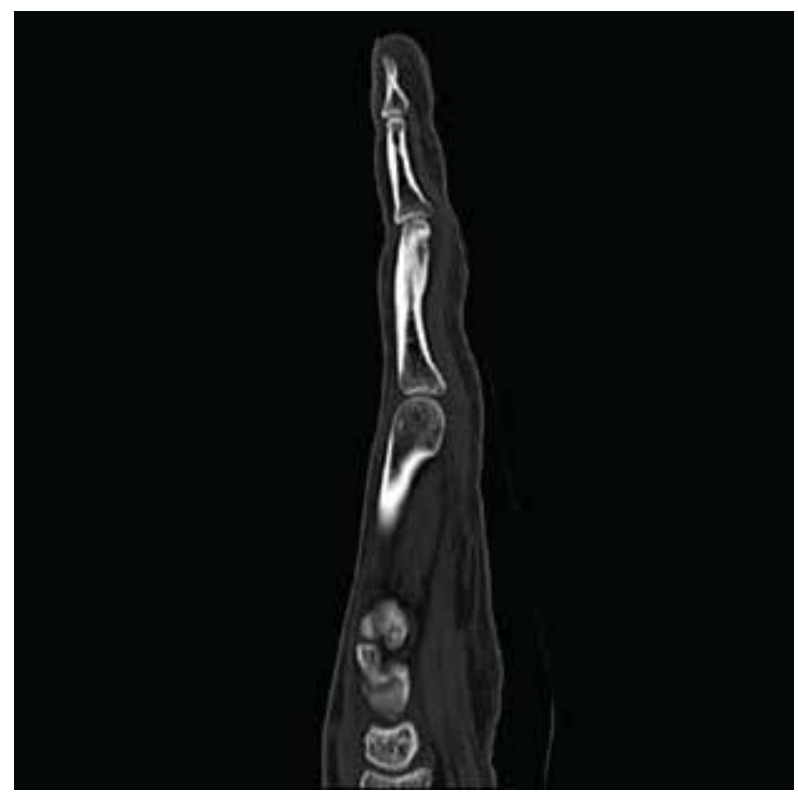

Fig. 1 (b)

Fig.1: CT scan of hand in (a) coronal and (b) sagittal plan demonstrate focal lytic area with sclerotic dot at the ulnar aspect of proximal phalanx near head (white arrow in a), associated surrounding sclerosis. The epicenter of the lesion could be periosteal. No periosteal new bone formation. Fusiform soft tissue swelling at the proximal phalanx noted. 
General condition and blood examination was normal. After a discussion with the patient and by consideration of his persistent pain and difficulty, it was decided to admit the patient and proceed with surgery.

The lesion was approached dorsally and exposed the pathology. Drill hole was made at the site of nidus and curettage was done. Tissue was sent for histopathology. Histopathology examination confirmed it as being an osteoid osteoma.

The patient was discharged the following day in a stable hemodynamic condition.

On follow up visit, the patient had no obvious pain which he was experiencing before the surgery.

\section{Discussion}

Occurrence of osteoid osteoma in the hand is unusual and in order of frequency, proximal, distal and middle phalanx is the most frequent site. Therefore, patients with this entity are often resumed to have other disorders more common to the region, delaying accurate diagnosis for an extended period of time. While the patient mentioned above did demonstrate the classic symptoms of osteoid osteoma, namely localized pain that is worse at night and relieved by NSAIDs, there have been reports of other patients with this bone lesion in the hand that had atypical symptoms. These include vague, diffuse as opposed to localized pain, fusiform as opposed to localized swelling of the digit, and decreased range of motion with periarticular lesions. ${ }^{5}$ Other authors have also reported presentations of profuse digital tip perspiration and intense autonomic phenomena, which may mimic peripheral nerve compression or reflex sympathetic dystrophy. ${ }^{6}$
Meng et al. reported the most common appearance of osteoid osteoma of the hand is of an eccentric lesion with soft tissue swelling and a relative absence of sclerosis, suggesting osteomyelitis. ${ }^{7}$ Most of the hand osteoid osteoma is diagnosed late because of atypical radiologic or clinical findings. This tumor may be misdiagnosed with conditions such as subacute osteomelitis, Brodie's abscess, tuberculosis, tenosynovitis.

Osteoid osteomas produce excrutiating pain that is disproportionate to their size; there are two postulated reasons for this. Within the nidus, nerve fibers are intimately associated with the blood vessels, and these fibers are larger and more numerous in the reactive zone. An excess of cyclooxygenases and prostoglandins within the lesion leads to vasodilation and also decreased nociceptive threshold of nerve endings resulting in sensations of pain. ${ }^{8}$ With the pharmacologic decrease in prostaglandin production, NSAIDs often provide predictable pain relief.

Patients with osteoid osteoma in the small bones of hand may present without pain or without a nidus or bone forming reaction and this is the reason for delay in diagnosis. Although this tumor may regress spontaneously after a long time, but surgery is usually indicated in symptomatic patients not responsive to medical treatment. A variety of treatment options including medication, wide excision of the nidus and curettage of the lesion, CT-guided core drill excision, radionuclide-guided excision, percutaneous radiofrequency ablation, injection of ethanol or interstitial laser photocoagulation (ILP) have been used for this tumor., ${ }^{4}$ Percutaneous CT-guided ablations with laser or 


\section{CASE REPORT}

radiofrequency have now widely replaced surgery as the treatment of choice for osteoid osteoma. However, surgical excision still plays a major role for the lesions in the hands and feet owing to the close relationship of the small bones with the neurovascular structures. ${ }^{8}$

\section{Conclusion}

Osteoid osteoma should always be considered in cases with phalangeal pain especially if the pain is worse at night time and subsides with NSAIDS. Computed tomography not only has the highest specificity but also allows for accurate preoperative planning.

\section{References}

1. Johnson T, Steinbach L. Essentials of musculoskeletal imaging. 1st ed. Illinois: American Academy of Orthopedic Surgeons; 2004.

2. Mirra J, Picci P, Gold R. Bone tumors: clinical, radiologic, and pathologic correlations. 2nd ed. Philadelphia: Lea and Febige;1989.

3. Galdi B, Capo JT, Nourbakhsh A, Patterson F. Osteoid osteoma of the thumb: a case report. Hand. 2010;5:423-26. 4. Heck RK. Benign bone tumors and non neoplastic condition simulation bone tumors. Terrycanale S, Beaty JH. Eds. Campbell's Operative Orthopedics, Mosby, Philadelphia. 2008: 855-57.

5. Levy Y, Rosenheck S, Greiff M, Torok G. Osteoid osteoma of the distal phalanx of the thumb. Acta Orthop Scand. 1979;50:667-69.

6. Nakatsuchi Y, Sugimoto Y, Nakano M. Osteoid osteoma of the terminal phalanx. J Hand Surg Br. 1984;9:201-3.

7. Meng Q, Watt L. Phalangeal Osteoid Osteoma. BJR. 1989;62:321-25.

8. Mungo DV, Zhang X, O'Keefe RJ, Rosier RN, Puzas JE, Schwarz EM. COX-1 and COX-2 expression in osteoid osteomas. J Orthop Res. 2002;20:159-62. 